Ashdin Publishing

Journal of Generalized Lie Theory and Applications

Vol. 4 (2010), Article ID S090601, 10 pages

doi:10.4303/jglta/S090601

\title{
Classifications of some classes of Zinbiel algebras
}

\author{
J. Q. ADASHEV, A. Kh. KHUDOYBERDIYEV, and B. A. OMIROV \\ Institute of Mathematics and Information Technologies of Academy of Sciences, \\ 29 F. Hodjaev Street, 100125 Tashkent, Uzbekistan \\ E-mails: adashevjq@mail.ru,khabror@mail.ru,omirovb@mail.ru
}

\begin{abstract}
In this work, the nul-filiform and filiform Zinbiel algebras are described up to isomorphism. Moreover, the classification of complex Zinbiel algebras dimensions $\leq 3$ is extended up to dimension 4.
\end{abstract}

2000 MSC: $17 \mathrm{~A} 32$

\section{Introduction}

One of the important objects of the modern theory of nonassociative algebras is Lie algebras. Active investigations in the theory of Lie algebras lead to the appearance of some generalizations of these algebras such as Mal'cev algebras, Lie superalgebras, binary Lie algebras, Leibniz algebras, and others.

In the present work, we consider algebras which are dual to Leibniz algebras. Recall that Leibniz algebras were introduced in [6] in the nineties of the last century. They are defined by the following identity:

$$
[x,[y, z]]=[[x, y], z]-[[x, z], y] .
$$

J.-L. Loday in [5] studied categorical properties of Leibniz algebras and considered in this connection a new object - Zinbiel algebra (read Leibniz in the reverse order). Since the category of Zinbiel algebras is Koszul dual to the category of Leibniz algebras, sometimes they are also called dual Leibniz algebras [7].

In works [3, 4], some interesting properties of Zinbiel algebras were obtained. In particular, the nilpotency of an arbitrary complex finite-dimensional Zinbiel algebra was proved in [4].

For the examples of Zinbiel algebras, we refer to works $[4,5,7]$.

Since description of all finite-dimensional complex Zinbiel algebras (which are nilpotent) is a boundless problem, it is natural to add certain restrictions for their investigation. One of such restrictions is the condition on the nilindex. Recall that in works $[2,8]$ some descriptions of nilpotent Leibniz algebras and Lie algebras are given.

At the beginning of the study of any class of algebras, it is important to describe up to isomorphism algebras of lower dimensions, because such description gives examples for to establish or reject certain conjectures. In this way, in [4], the classification of complex Zinbiel algebras of dimensions $\leq 3$ is given. Applying some general results obtained for finite-dimensional Zinbiel algebras, we extend the classification of complex Zinbiel algebras up to dimension 4. It should be noted that this classification shows that associative algebras play the crucial role in the classification of four-dimensional algebras, which are defined by the multilinear identity of degree 3 . 


\section{Classification of complex nul-filiform Zinbiel algebras}

Definition 2.1. An algebra $A$ over a field $F$ is called Zinbiel algebra if for any $x, y, z \in A$ the identity

$$
(x \circ y) \circ z=x \circ(y \circ z)+x \circ(z \circ y)
$$

holds.

For a given Zinbiel algebra $A$, we define the following sequence:

$$
A^{1}=A, \quad A^{k+1}=A \circ A^{k}, \quad k \geq 1 .
$$

Definition 2.2. A Zinbiel algebra $A$ is called nilpotent if there exists $s \in N$ such that $A^{s}=0$. The minimal number $s$ satisfying this property is called index of nilpotency or nilindex of the algebra $A$.

It is not difficult to see that the index of nilpotency of an arbitrary $n$-dimensional nilpotent algebra does not exceed the number $n+1$.

Definition 2.3. An $n$-dimensional Zinbiel algebra $A$ is called nul-filiform if $\operatorname{dim} A^{i}=$ $(n+1)-i, 1 \leq i \leq n+1$.

It is evident that the last definition is equivalent to the fact that algebra $A$ has maximal index of nilpotency.

Theorem 2.4. An arbitrary $n$-dimensional nul-filiform Zinbiel algebra is isomorphic to the following algebra:

$$
e_{i} \circ e_{j}=C_{i+j-1}^{j} e_{i+j}, \quad \text { for } 2 \leq i+j \leq n,
$$

where omitted products are equal to zero and $\left\{e_{1}, e_{2}, \ldots, e_{n}\right\}$ is a basis of the algebra, the symbols $C_{s}^{t}$ are binomial coefficients defined as $C_{s}^{t}=\frac{s !}{t !(s-t !)}$.

Proof. Let $A$ be an $n$-dimensional nul-filiform Zinbiel algebra and let $\left\{x_{1}, x_{2}, \ldots, x_{n}\right\}$ be a basis of the algebra $A$ such that $x_{1} \in A^{1} \backslash A^{2}, x_{2} \in A^{2} \backslash A^{3}, \ldots, x_{n} \in A^{n}$. Since $x_{2} \in A^{2} \backslash A^{3}$, for some elements $b_{2, p}, c_{2, p}$ of algebra $A$, we have

$$
x_{2}=\sum b_{2, p} \circ c_{2, p}=\sum \alpha_{i, j} x_{i} \circ x_{j}=\alpha_{1,1} x_{1} \circ x_{1}+(*),
$$

where $(*) \in A^{3}$, i.e., $x_{2}=\alpha_{1,1} x_{1} \circ x_{1}+(*)$. Note that $\alpha_{1,1} x_{1} \circ x_{1} \neq 0$. Indeed, in the opposite case, $x_{2} \in A^{3}$.

Similarly, for $x_{3} \in A^{3} \backslash A^{4}$, we have

$$
x_{3}=\sum a_{3, p} \circ\left(b_{3, p} \circ c_{3, p}\right)=\sum \alpha_{i, j, k} x_{i} \circ\left(x_{j} \circ x_{k}\right)=\alpha_{1,1,1} x_{1} \circ\left(x_{1} \circ x_{1}\right)+(* *),
$$

where $(* *) \in A^{4}$ and $\alpha_{1,1,1} x_{1} \circ\left(x_{1} \circ x_{1}\right) \neq 0$ (otherwise $\left.x_{3} \in A^{4}\right)$, i.e., $x_{3}=\alpha_{1,1,1} x_{1} \circ$ $\left(x_{1} \circ x_{1}\right)+(* *)$. Continuing this process, we obtain that elements

$$
e_{1}:=x_{1}, \quad e_{2}:=x_{1} \circ x_{1}, \quad e_{3}:=x_{1} \circ\left(x_{1} \circ x_{1}\right), \quad \ldots, \quad e_{n}:=\left(x_{1} \circ \cdots \circ\left(x_{1} \circ\left(x_{1} \circ x_{1}\right)\right)\right)
$$


are distinct from zero. It is not difficult to check the linear independence of these elements. Hence, we can choose the elements $\left\{e_{1}, e_{2}, \ldots, e_{n}\right\}$ as a basis of algebra $A$. We have by construction

$$
e_{1} \circ e_{i}=e_{i+1} \quad \text { for } 1 \leq i \leq n-1 .
$$

We prove equality (2.2) by induction on $j$ for every $i$.

Using identities (2.1), (2.3), we can prove by induction the equality

$$
e_{i} \circ e_{1}=i e_{i+1} \quad \text { for } 1 \leq i \leq n-1,
$$

i.e., equality (2.2) is true for $j=1$ and every $i$.

Suppose that the equality is true for all $j \leq k-1$ and every $i$.

Let us prove the equality (2.2) for $j=k$ and every $i$. Using the inductive hypothesis and the following chain of equalities:

$$
\begin{aligned}
e_{i} \circ e_{k} & =e_{i} \circ\left(e_{1} \circ e_{k-1}\right)=\left(e_{i} \circ e_{1}\right) \circ e_{k-1}-e_{i} \circ\left(e_{k-1} \circ e_{1}\right) \\
& =i e_{i+1} \circ e_{k-1}-(k-1) e_{i} \circ e_{k}=i C_{i+k-1}^{k-1} e_{i+k}-(k-1) e_{i} \circ e_{k},
\end{aligned}
$$

we obtain $k e_{i} \circ e_{k}=i C_{i+k-1}^{k-1} e_{i+k}$, i.e.,

$$
e_{i} \circ e_{k}=\frac{i}{k} C_{i+k-1}^{k-1} e_{i+k}=\frac{i}{k} \frac{(i+k-1) !}{(k-1) ! i !} e_{i+k}=\frac{(i+k-1) !}{k !(i-1) !} e_{i+k}=C_{i+k-1}^{k} e_{i+k} .
$$

We denote the algebra from Theorem 2.4 as $N F_{n}$. It is not difficult to see that the $n$ dimensional Zinbiel algebra is one generated if and only if it is isomorphic to the algebra $N F_{n}$.

\section{Classification of complex filiform Zinbiel algebras}

In this section, we classify filiform Zinbiel algebras.

Definition 3.1. An $n$-dimensional Zinbiel algebra $A$ is said to be filiform if $\operatorname{dim} A^{i}=n-i$, $2 \leq i \leq n$.

Definition 3.2. Given a filiform Zinbiel algebra $A$, put $A_{i}=A^{i} / A^{i+1}, 1 \leq i \leq n-1$ and $\operatorname{grA}=A_{1} \oplus A_{2} \oplus \cdots \oplus A_{n-1}$. Then $A_{i} \circ A_{j} \subseteq A_{i+j}, \operatorname{dim} A_{1}=2, \operatorname{dim} A_{i}=1$ for $2 \leq i \leq n-1$ and we obtain the graded algebra grA. If an algebra $B$ is isomorphic to grA, then we say that the algebra $B$ is naturally graded.

In the following theorem, the classification of complex naturally graded filiform Zinbiel algebras is represented.

Theorem 3.3. An arbitrary $n$-dimensional $(n \geq 5)$ naturally graded complex filiform Zinbiel algebra is isomorphic to the following algebra:

$$
F_{n}: e_{i} \circ e_{j}=C_{i+j-1}^{j} e_{i+j}, \quad 2 \leq i+j \leq n-1,
$$

where omitted products are equal to zero and $\left\{e_{1}, e_{2}, \ldots, e_{n}\right\}$ is a basis of the algebra.

Proof. Let $A$ be a Zinbiel algebra satisfying conditions of the theorem. Similar to the work [8], we choose a basis $\left\{e_{1}, e_{2}, \ldots, e_{n}\right\}$ of the algebra $A$ such that $A_{1}=\left\langle e_{1}, e_{n}\right\rangle, A_{j}=\left\langle e_{j}\right\rangle$, $2 \leq j \leq n-1$ and $e_{1} \circ e_{i}=e_{i+1}$ for $2 \leq i \leq n-2$. 
Introduce the following notations:

$e_{1} \circ e_{1}=\alpha_{1} e_{2}, \quad e_{1} \circ e_{n}=\alpha_{2} e_{2}, \quad e_{n} \circ e_{1}=\alpha_{3} e_{2}, \quad e_{n} \circ e_{n}=\alpha_{4} e_{2}$.

Consider the possible cases.

Case 1. Let $\left(\alpha_{1}, \alpha_{4}\right) \neq(0,0)$. Then without loss of generality we can suppose $\alpha_{1} \neq 0$. Change the basis as follows: $e_{1}^{\prime}=e_{1}, e_{i}^{\prime}=\alpha_{1} e_{i}, 2 \leq i \leq n-1$. We can suppose $\alpha_{1}=1$. Then the space spanned on the vectors $\left\{e_{1}, e_{2}, \ldots, e_{n-1}\right\}$ forms a nul-filiform Zinbiel algebra of the dimension $n-1$. From the proof of Theorem 2.4, we can conclude $e_{i} \circ e_{j}=C_{i+j-1}^{j} e_{i+j}$, $2 \leq i+j \leq n-1$.

Let us show that the omitted products are equal to zero.

Apply identity (2.1) in the following multiplications:

$$
\left(e_{1} \circ e_{n}\right) \circ e_{1}=e_{1} \circ\left(e_{n} \circ e_{1}\right)+e_{1} \circ\left(e_{1} \circ e_{n}\right) \Longrightarrow 2 \alpha_{2} e_{3}=\alpha_{3} e_{3}+\alpha_{2} e_{3},
$$

i.e., $\alpha_{2}=\alpha_{3}$;

$$
\left(e_{1} \circ e_{1}\right) \circ e_{n}=e_{1} \circ\left(e_{1} \circ e_{n}\right)+e_{1} \circ\left(e_{n} \circ e_{1}\right) \Longrightarrow e_{2} \circ e_{n}=\alpha_{2} e_{3}+\alpha_{3} e_{3},
$$

hence, $e_{2} \circ e_{n}=2 \alpha_{2} e_{3}$;

$$
\left(e_{1} \circ e_{n}\right) \circ e_{n}=2 e_{1} \circ\left(e_{n} \circ e_{n}\right) \Longrightarrow 2 \alpha_{2}^{2} e_{3}=2 \alpha_{4} e_{3},
$$

consequently, $\alpha_{2}^{2}=\alpha_{4}$;

$$
\left(e_{n} \circ e_{1}\right) \circ e_{1}=2 e_{n} \circ\left(e_{1} \circ e_{1}\right) \Longrightarrow 2 \alpha_{2} e_{3}=2 e_{n} \circ e_{2},
$$

i.e., we have $e_{n} \circ e_{2}=\alpha_{2} e_{3}$.

Taking the change of the basic elements by the following way:

$$
e_{n}^{\prime}=-\alpha_{2} e_{1}+e_{n}, \quad e_{i}^{\prime}=e_{i} \quad \text { for } 1 \leq i \leq n-1,
$$

it is not difficult to see that $e_{1}^{\prime} \circ e_{n}^{\prime}=e_{n}^{\prime} \circ e_{1}^{\prime}=e_{n}^{\prime} \circ e_{n}^{\prime}=e_{2}^{\prime} \circ e_{n}^{\prime}=e_{n}^{\prime} \circ e_{2}^{\prime}=0$. Moreover, the other products are not changed, i.e., we can suppose $\alpha_{2}=\alpha_{3}=\alpha_{4}=0$.

Using identity (2.1) and the method of mathematical induction, it is easy to prove

$$
e_{n} \circ e_{i}=0 \quad \text { for } 1 \leq i \leq n-1 .
$$

The equality

$$
e_{i} \circ e_{n}=0 \quad \text { for } 1 \leq i \leq n-1
$$

can be proved by induction and using (2.1), (3.1).

Case 2. Let $\left(\alpha_{1}, \alpha_{4}\right)=(0,0)$. Then $\left(\alpha_{2}, \alpha_{3}\right) \neq(0,0)$. In the case of $\alpha_{2} \neq-\alpha_{3}$, taking $e_{1}^{\prime}=e_{1}+e_{n}$, we obtain the conditions of Case 1 . Therefore, we need to consider only the case of $\alpha_{2}=-\alpha_{3} \neq 0$. By the following change of basis:

$$
e_{1}^{\prime}=e_{1}, \quad e_{n}^{\prime}=e_{n}, \quad e_{i}^{\prime}=\alpha_{2} e_{i} \quad \text { for } 2 \leq i \leq n-1,
$$

we can suppose $\alpha_{2}=1$.

The products

$$
\left(e_{1} \circ e_{n}\right) \circ e_{1}=e_{1} \circ\left(e_{n} \circ e_{1}\right)+e_{1} \circ\left(e_{1} \circ e_{n}\right) \Longrightarrow e_{2} \circ e_{1}=0,
$$




$$
0=\left(e_{1} \circ e_{1}\right) \circ e_{2}=e_{1} \circ\left(e_{1} \circ e_{2}\right)+e_{1} \circ\left(e_{2} \circ e_{1}\right)=e_{1} \circ e_{3}=e_{4}
$$

deduce the contradiction to the existence of algebra in this case.

The following proposition allows to extract a "convenient" basis in an arbitrary complex filiform Zinbiel algebra. Such basis in the literature is called adapted [8].

Proposition 3.4. There exists a basis $\left\{e_{1}, e_{2}, \ldots, e_{n}\right\}$ in an arbitrary $n$-dimensional $(n \geq 5)$ complex filiform Zinbiel algebra such that the multiplication of the algebra has the following form:

$$
\begin{aligned}
& e_{i} \circ e_{j}=C_{i+j-1}^{j} e_{i+j}, \quad 2 \leq i+j \leq n-1, \\
& e_{n} \circ e_{1}=\alpha e_{n-1}, \quad e_{n} \circ e_{n}=\beta e_{n-1},
\end{aligned}
$$

where $\alpha, \beta \in \mathbb{C}$.

Proof. By Theorem 3.3 we have that any $n$-dimensional complex filiform Zinbiel algebra is isomorphic to the algebra of the form $F_{n}+\beta$, where

$$
\begin{aligned}
& \beta\left(e_{1}, e_{i}\right)=0 \text { for } 1 \leq i \leq n-1, \\
& \beta\left(e_{n}, e_{n}\right) \in \operatorname{lin}\left\{e_{3}, e_{4}, \ldots, e_{n-1}\right\}, \\
& \beta\left(e_{i}, e_{n}\right), \beta\left(e_{n}, e_{i}\right) \in \operatorname{lin}\left\{e_{i+2}, e_{i+3}, \ldots, e_{n-1}\right\} \quad \text { for } 1 \leq i \leq n-1, \\
& \beta\left(e_{i}, e_{1}\right) \in \operatorname{lin}\left\{e_{i+2}, e_{i+3}, \ldots, e_{n-1}\right\} \quad \text { for } 2 \leq i \leq n-1, \\
& \beta\left(e_{i}, e_{j}\right) \in \operatorname{lin}\left\{e_{i+j+1}, e_{i+j+2}, \ldots, e_{n-1}\right\} \quad \text { for } 2 \leq i, j \leq n-1 .
\end{aligned}
$$

Similarly to the proof of Theorem 3.3, it is not difficult to establish that the multiplications

$$
e_{i} \circ e_{1}, \quad e_{i} \circ e_{j}, \quad 1 \leq i, j \leq n-1
$$

can be obtained from $e_{1} \circ e_{i}=e_{i+1}, 1 \leq i \leq n-2$, and identity (2.1).

By the similar process, we obtain

$$
\beta\left(e_{i}, e_{1}\right)=0 \quad \text { for } 1 \leq i \leq n-1, \quad \beta\left(e_{i}, e_{j}\right)=0 \quad \text { for } 2 \leq i, j \leq n-1 .
$$

So, we have the products

$$
e_{i} \circ e_{j}=C_{i+j-1}^{j} e_{i+j}, \quad 2 \leq i+j \leq n-1 .
$$

Now define the products $e_{i} \circ e_{n}$ and $e_{n} \circ e_{i}$ for $1 \leq i \leq n$ and put

$$
e_{1} \circ e_{n}=\alpha_{3} e_{3}+\alpha_{4} e_{4}+\cdots+\alpha_{n-1} e_{n-1}, \quad e_{n} \circ e_{1}=\beta_{3} e_{3}+\beta_{4} e_{4}+\cdots+\beta_{n-1} e_{n-1} .
$$

Taking the change

$$
e_{n}^{\prime}=e_{n}-\alpha_{3} e_{2}-\alpha_{4} e_{3}-\cdots-\alpha_{n-1} e_{n-2},
$$

we can suppose $\alpha_{3}=\alpha_{4}=\cdots=\alpha_{n-1}=0$, i.e., $e_{1} \circ e_{n}=0$.

Identity (2.1) implies

$$
\left(e_{1} \circ e_{n}\right) \circ e_{1}=e_{1} \circ\left(e_{n} \circ e_{1}\right)+e_{1} \circ\left(e_{1} \circ e_{n}\right) \Longrightarrow e_{1} \circ\left(\beta_{3} e_{3}+\beta_{4} e_{4}+\cdots+\beta_{n-1} e_{n-1}\right)=0 .
$$

Therefore, $\beta_{3} e_{4}+\beta_{4} e_{5}+\cdots+\beta_{n-2} e_{n-1}=0$ and $e_{n} \circ e_{1}=\beta_{n-1} e_{n-1}$. 
Consider the product

$$
\left(e_{n} \circ e_{n}\right) \circ e_{1}=e_{n} \circ\left(e_{n} \circ e_{1}\right)+e_{n} \circ\left(e_{1} \circ e_{n}\right)=0
$$

from which we obtain $e_{n} \circ e_{n}=\gamma e_{n-1}$ for some $\gamma$.

Similar to the proof of Theorem 3.3, we can obtain that $e_{n} \circ e_{i}=0$ and $e_{i} \circ e_{n}=0$ for $2 \leq i \leq n-1$, which complete the proof of the proposition.

The classification of complex filiform Zinbiel algebras is given in the following theorem.

Theorem 3.5. An arbitrary $n$-dimensional $(n \geq 5)$ complex filiform Zinbiel algebra is isomorphic to one of the following pairwise nonisomorphic algebras:

$$
\begin{array}{lll}
F_{n}^{1}: e_{i} \circ e_{j}=C_{i+j-1}^{j} e_{i+j}, & 2 \leq i+j \leq n-1 ; & \\
F_{n}^{2}: e_{i} \circ e_{j}=C_{i+j-1}^{j} e_{i+j}, & 2 \leq i+j \leq n-1, & e_{n} \circ e_{1}=e_{n-1} ; \\
F_{n}^{3}: e_{i} \circ e_{j}=C_{i+j-1}^{j} e_{i+j}, & 2 \leq i+j \leq n-1, & e_{n} \circ e_{n}=e_{n-1} .
\end{array}
$$

Proof. From Proposition 3.4, we have the multiplication (multiplication (3.2)) of $n$ dimensional complex filiform Zinbiel algebra, namely,

$$
\begin{aligned}
& e_{i} \circ e_{j}=C_{i+j-1}^{j} e_{i+j}, \quad 2 \leq i+j \leq n-1, \\
& e_{n} \circ e_{1}=\alpha e_{n-1}, \quad e_{n} \circ e_{n}=\beta e_{n-1} .
\end{aligned}
$$

Let us check the isomorphism inside this family of algebras. Consider the general change of the generators of the basic elements in the form

$$
e_{1}^{\prime}=a_{1} e_{1}+a_{2} e_{2}+\cdots+a_{n} e_{n}, \quad e_{n}^{\prime}=b_{1} e_{1}+b_{2} e_{2}+\cdots+b_{n} e_{n},
$$

where $a_{1} \neq 0$ and $a_{1} b_{n}-a_{n} b_{1} \neq 0$. Then for the remainder elements of the new basis we have

$$
e_{2}^{\prime}=a_{1}^{2} e_{2}+h_{3}, \quad e_{3}^{\prime}=a_{1}^{3} e_{3}+h_{4}, \quad \ldots, \quad e_{n-1}^{\prime}=a_{1}^{n-1} e_{n-1},
$$

where $h_{i} \in A^{i}$. The relations

$$
e_{i}^{\prime} \circ e_{j}^{\prime}=C_{i+j-1}^{j} e_{i+j}^{\prime}, \quad 2 \leq i+j \leq n-1,
$$

imply the following restrictions:

$$
\begin{aligned}
& a_{1} b_{1}=0, \\
& a_{1} b_{2}+2 a_{2} b_{1}=0, \\
& a_{1} b_{3}+C_{3}^{2} a_{2} b_{2}+3 a_{3} b_{1}=0, \\
& \quad \vdots \\
& a_{1} b_{n-3}+C_{n-3}^{n-4} a_{2} b_{n-4}+C_{n-3}^{n-5} a_{3} b_{n-5}+\cdots+(n-3) a_{n-3} b_{1}=0, \\
& a_{1} b_{n-2}+C_{n-2}^{n-3} a_{2} b_{n-3}+C_{n-2}^{n-4} a_{3} b_{n-4}+\cdots+(n-2) a_{n-2} b_{1}+\alpha a_{n} b_{1}+\beta a_{n} b_{n}=0 .
\end{aligned}
$$

From these restrictions, we get

$$
b_{1}=b_{2}=\cdots=b_{n-3}=0, \quad b_{n-2}=-\frac{a_{n} b_{n}}{a_{1}} \beta .
$$


Consider the product

$$
\begin{aligned}
e_{n}^{\prime} \circ e_{1}^{\prime} & =\left(-\frac{a_{n} b_{n}}{a_{1}} \beta e_{n-2}+b_{n-1} e_{n-1}+b_{n} e_{n}\right) \circ\left(a_{1} e_{1}+a_{2} e_{2}+\cdots+a_{n} e_{n}\right) \\
& =-(n-2) \beta a_{n} b_{n} e_{n-1}+\alpha a_{1} b_{n} e_{n-1}+\beta a_{n} b_{n} e_{n-1}=\left(\alpha a_{1} b_{n}-(n-3) a_{n} b_{n}\right) e_{n-1} .
\end{aligned}
$$

On the other hand,

$$
e_{n}^{\prime} \circ e_{1}^{\prime}=\alpha^{\prime} e_{n-1}^{\prime}=\alpha^{\prime} a_{1}^{n-1} e_{n-1} .
$$

Comparing the coefficients at the basic element $e_{n-1}$, we obtain

$$
\alpha a_{1} b_{n}-(n-3) \beta a_{n} b_{n}=\alpha^{\prime} a_{1}^{n-1} .
$$

Consider the product

$$
\begin{aligned}
e_{n}^{\prime} \circ e_{n}^{\prime} & =\left(-\frac{a_{n} b_{n}}{a_{1}} \beta e_{n-2}+b_{n-1} e_{n-1}+b_{n} e_{n}\right) \circ\left(-\frac{a_{n} b_{n}}{a_{1}} \beta e_{n-2}+b_{n-1} e_{n-1}+b_{n} e_{n}\right) \\
& =b_{n}^{2} \beta e_{n-1} .
\end{aligned}
$$

On the other hand,

$$
e_{n}^{\prime} \circ e_{n}^{\prime}=\beta^{\prime} e_{n-1}^{\prime}=\beta^{\prime} a_{1}^{n-1} e_{n-1} .
$$

Comparing the coefficients, we have

$$
b_{n}^{2} \beta=\beta^{\prime} a_{1}^{n-1} .
$$

Now consider the following cases.

Case 1. Let $\beta=0$. Then $\beta^{\prime}=0$ and $\alpha b_{n}=\alpha^{\prime} a_{1}^{n-2}$. If $\alpha=0$, then $\alpha^{\prime}=0$ and we have algebra $F_{n}^{1}$. If $\alpha \neq 0$, then taking $b_{n}=\frac{a_{1}^{n-2}}{\alpha}$ we get $\alpha^{\prime}=1$, i.e., the algebra $F_{n}^{2}$ is obtained.

Case 2. Let $\beta \neq 0$. Then putting $b_{n}=\sqrt{\frac{a_{1}^{n-1}}{\beta}}, a_{n}=\frac{\alpha a_{1}}{(n-3) \beta}$ we get $\beta^{\prime}=1, \alpha^{\prime}=0$ and algebra $F_{n}^{3}$ is obtained.

Note that the obtained algebras are not pairwise isomorphic.

Comparing the description of complex filiform Leibniz algebras [2] and the result of Theorem 3.5, we can note how the class of complex filiform Zinbiel algebras is "thinner". So, although Zinbiel algebras and Leibniz algebras are Koszul dual, they are quantitatively strongly distinguished even in the class of filiform algebras.

It is known that for any variety of algebras $\mathbb{Z}_{2}$-graded algebras of that variety can be defined, which is called superalgebras. In the same way, we define a notion of Zinbiel superalgebra $A=A_{0} \oplus A_{1}$ by the following identity:

$$
(x \circ y) \circ z=x \circ(y \circ z)+(-1)^{\alpha \beta} x \circ(z \circ y),
$$

where $y \in A_{\alpha}, z \in A_{\beta}$ and $\alpha, \beta \in \mathbb{Z}_{2}$.

It should be noted that the proof on the nilpotency of finite-dimensional complex Zinbiel algebras can be extended to the proof of solvability of the finite-dimensional complex Zinbiel superalgebras.

Moreover, from the obtained classification of complex filiform Zinbiel algebras, we give the following conjecture.

Conjecture 3.6. Finite-dimensional complex $\mathbb{Z}_{2}$-graded Zinbiel algebra (Zinbiel superalgebra) is nilpotent. 


\section{Classification of four-dimensional complex Zinbiel algebras}

Since an arbitrary finite-dimensional complex Zinbiel algebra is nilpotent, then for an arbitrary four-dimensional Zinbiel algebra $A$ the condition $A^{5}=0$ holds.

Take into account that the direct sum of nilpotent Zinbiel algebras is nilpotent; further we will not consider split algebras case.

Theorem 4.1. An arbitrary four-dimensional complex nonsplit Zinbiel algebra is isomorphic to the one of the following pairwise nonisomorphic algebras:

$$
\begin{aligned}
& A_{1}: e_{1} \circ e_{1}=e_{2}, e_{1} \circ e_{2}=e_{3}, e_{2} \circ e_{1}=2 e_{3}, e_{1} \circ e_{3}=e_{4}, e_{2} \circ e_{2}=3 e_{4}, e_{3} \circ e_{1}=3 e_{4} ; \\
& A_{2}: e_{1} \circ e_{1}=e_{3}, e_{1} \circ e_{2}=e_{4}, e_{1} \circ e_{3}=e_{4}, e_{3} \circ e_{1}=2 e_{4} ; \\
& A_{3}: e_{1} \circ e_{1}=e_{3}, e_{1} \circ e_{3}=e_{4}, e_{2} \circ e_{2}=e_{4}, e_{3} \circ e_{1}=2 e_{4} ; \\
& A_{4}: e_{1} \circ e_{2}=e_{3}, e_{1} \circ e_{3}=e_{4}, e_{2} \circ e_{1}=-e_{3} ; \\
& A_{5}: e_{1} \circ e_{2}=e_{3}, e_{1} \circ e_{3}=e_{4}, e_{2} \circ e_{1}=-e_{3}, e_{2} \circ e_{2}=e_{4} ; \\
& A_{6}: e_{1} \circ e_{1}=e_{4}, e_{1} \circ e_{2}=e_{3}, e_{2} \circ e_{1}=-e_{3}, e_{2} \circ e_{2}=-2 e_{3}+e_{4} ; \\
& A_{7}: e_{1} \circ e_{2}=e_{3}, e_{2} \circ e_{1}=e_{4}, e_{2} \circ e_{2}=-e_{3} ; \\
& A_{8}(\alpha): e_{1} \circ e_{1}=e_{3}, e_{1} \circ e_{2}=e_{4}, e_{2} \circ e_{1}=-\alpha e_{3}, e_{2} \circ e_{2}=-e_{4}, \alpha \in \mathbb{C} ; \\
& A_{9}(\alpha): e_{1} \circ e_{1}=e_{4}, e_{1} \circ e_{2}=\alpha e_{4}, e_{2} \circ e_{1}=-\alpha e_{4}, e_{2} \circ e_{2}=e_{4}, e_{3} \circ e_{3}=e_{4}, \alpha \in \mathbb{C} ; \\
& A_{10}: e_{1} \circ e_{2}=e_{4}, e_{1} \circ e_{3}=e_{4}, e_{2} \circ e_{1}=-e_{4}, e_{2} \circ e_{2}=e_{4}, e_{3} \circ e_{1}=e_{4} ; \\
& A_{11}: e_{1} \circ e_{1}=e_{4}, e_{1} \circ e_{2}=e_{4}, e_{2} \circ e_{1}=-e_{4}, e_{3} \circ e_{3}=e_{4} ; \\
& A_{12}: e_{1} \circ e_{2}=e_{3}, e_{2} \circ e_{1}=e_{4} ; \\
& A_{13}: e_{1} \circ e_{2}=e_{3}, e_{2} \circ e_{1}=-e_{3}, e_{2} \circ e_{2}=e_{4} ; \\
& A_{14}: e_{2} \circ e_{1}=e_{4}, e_{2} \circ e_{2}=e_{3} ; \\
& A_{15}(\alpha): e_{1} \circ e_{2}=e_{4}, e_{2} \circ e_{2}=e_{3}, e_{2} \circ e_{1}=\frac{1+\alpha}{1-\alpha} e_{4}, \alpha \in \mathbb{C} \backslash\{1\} ; \\
& A_{16}: e_{1} \circ e_{2}=e_{4}, e_{2} \circ e_{1}=-e_{4}, e_{3} \circ e_{3}=e_{4} .
\end{aligned}
$$

Proof. Note that the result of [1, Proposition 3.1] also holds for Zinbiel algebras. Therefore, we have the following possible cases for invariant sequence $\left(\operatorname{dim} A^{2}, \operatorname{dim} A^{3}, \operatorname{dim} A^{4}\right)$ :

$$
(3,2,1),(2,1,0),(2,0,0),(1,0,0),(0,0,0) \text {. }
$$

Obviously, a Zinbiel algebra with the condition $(3,2,1)$ is nul-filiform. Using Theorem 2.4, we obtain the algebra $A_{1}$.

Consider an algebra with the invariant sequence $(2,1,0)$ (this algebra is filiform).

Let $\left\{e_{1}, e_{2}, e_{3}, e_{4}\right\}$ be a basis of algebra $A$ satisfying the conditions $A^{2}=\left\{e_{3}, e_{4}\right\}, A^{3}=$ $\left\{e_{4}\right\}$. Then we can suppose that

$$
\begin{aligned}
& e_{1} \circ e_{1}=\alpha_{1} e_{3}+\alpha_{2} e_{4}, \quad e_{1} \circ e_{2}=\alpha_{3} e_{3}+\alpha_{4} e_{4}, \quad e_{2} \circ e_{1}=\alpha_{5} e_{3}+\alpha_{6} e_{4}, \\
& e_{2} \circ e_{2}=\alpha_{7} e_{3}+\alpha_{8} e_{4}, \quad e_{1} \circ e_{3}=e_{4}, \quad e_{2} \circ e_{3}=\alpha_{9} e_{4},
\end{aligned}
$$

where $\left(\alpha_{1}, \alpha_{3}, \alpha_{5}, \alpha_{7}\right) \neq(0,0,0,0)$.

Case 1. Let $\left(\alpha_{1}, \alpha_{7}\right) \neq(0,0)$. Then by arguments analogous to the arguments in the proofs of Theorems 3.3 and 3.5 we obtain the following algebras:

$$
e_{1} \circ e_{1}=e_{3}, \quad e_{1} \circ e_{3}=e_{4}, \quad e_{3} \circ e_{1}=2 e_{4}
$$




$$
\begin{aligned}
& e_{1} \circ e_{1}=e_{3}, \quad e_{1} \circ e_{2}=e_{4}, \quad e_{1} \circ e_{3}=e_{4}, \quad e_{3} \circ e_{1}=2 e_{4} ; \\
& e_{1} \circ e_{1}=e_{3}, \quad e_{1} \circ e_{3}=e_{4}, \quad e_{2} \circ e_{2}=e_{4}, \quad e_{3} \circ e_{1}=2 e_{4} .
\end{aligned}
$$

Note that the algebra defined by multiplication

$$
e_{1} \circ e_{1}=e_{3}, \quad e_{1} \circ e_{3}=e_{4}, \quad e_{3} \circ e_{1}=2 e_{4}
$$

is split. So, in this case, we have the algebras $A_{2}, A_{3}$.

Case 2. Let $\left(\alpha_{1}, \alpha_{7}\right)=(0,0)$. Then $\left(\alpha_{3}, \alpha_{5}\right) \neq(0,0)$. If $\alpha_{3} \neq-\alpha_{5}$, then taking $e_{1}^{\prime}=A e_{1}+e_{2}$, where $A \neq-\alpha_{9}$, we have Case 1 . It remains to consider the case $\alpha_{3}=-\alpha_{5}$. Denote $e_{3}^{\prime}=$ $\alpha_{3} e_{3}+\alpha_{4} e_{4}$. Then we can write

$$
\begin{aligned}
& e_{1} \circ e_{1}=\alpha_{2} e_{4}, \quad e_{1} \circ e_{2}=e_{3}, \quad e_{2} \circ e_{1}=-e_{3}+\alpha_{6} e_{4}, \\
& e_{2} \circ e_{2}=\alpha_{8} e_{4}, \quad e_{1} \circ e_{3}=e_{4}, \quad e_{2} \circ e_{3}=\alpha_{9} e_{4} .
\end{aligned}
$$

Consider the products

$$
\begin{aligned}
\left(e_{1} \circ e_{2}\right) \circ e_{1} & =e_{1} \circ\left(e_{2} \circ e_{1}\right)+e_{1} \circ\left(e_{1} \circ e_{2}\right)=e_{1} \circ\left(-e_{3}+\alpha_{6} e_{4}\right)+e_{1} \circ e_{3} \\
& =0 \Longrightarrow e_{3} \circ e_{1}=0, \\
\left(e_{1} \circ e_{2}\right) \circ e_{2} & =2 e_{1} \circ\left(e_{2} \circ e_{2}\right)=0 \Longrightarrow e_{3} \circ e_{2}=0 .
\end{aligned}
$$

If we replace basic elements as follows:

$$
e_{1}^{\prime}=e_{1}, \quad e_{2}^{\prime}=e_{2}-\alpha_{9} e_{1}, \quad e_{3}^{\prime}=e_{3}-\alpha_{2} \alpha_{9} e_{4}, \quad e_{4}^{\prime}=e_{4},
$$

we obtain $\alpha_{9}=0$, i.e., the multiplication in the algebra has the following form:

$$
e_{1} \circ e_{1}=\alpha e_{4}, \quad e_{1} \circ e_{2}=e_{3}, \quad e_{2} \circ e_{1}=-e_{3}+\beta e_{4}, \quad e_{2} \circ e_{2}=\gamma e_{4}, \quad e_{1} \circ e_{3}=e_{4}
$$

(omitted products are equal to zero).

Let us check the isomorphism inside this family.

Consider the general change of generators of the basic elements:

$$
e_{1}^{\prime}=a_{1} e_{1}+a_{2} e_{2}+a_{3} e_{3}, \quad e_{2}^{\prime}=b_{1} e_{1}+b_{2} e_{2}+b_{3} e_{3}
$$

where $a_{1} b_{2}-a_{2} b_{1} \neq 0$. Expressing basic elements $\left\{e_{3}^{\prime}, e_{4}^{\prime}\right\}$ via basic elements $\left\{e_{1}, e_{2}, e_{3}, e_{4}\right\}$ and analyzing the relations of the family in new basis, we obtain the following restrictions:

$$
\begin{aligned}
& a_{1}^{2} \alpha+a_{1} a_{2} \beta+a_{2}^{2} \gamma+a_{1} a_{3}=\alpha^{\prime} a_{1}^{2} b_{2}, \quad a_{1} b_{2} \beta+2 a_{2} b_{2} \gamma+a_{1} b_{3}=\beta^{\prime} a_{1}^{2} b_{2}, \\
& b_{2} \gamma=\gamma^{\prime} a_{1}^{2}, \quad b_{1}=0 .
\end{aligned}
$$

Consider the following cases.

Case 2.1. Let $\gamma=0$. Then $\gamma^{\prime}=0$ and

$$
a_{1} \alpha+a_{2} \beta+a_{3}=\alpha^{\prime} a_{1} b_{2}, \quad b_{2}+b_{3}=\beta^{\prime} a_{1} b_{2} .
$$

Taking $a_{3}=-a_{1} \alpha-a_{2} \beta$ and $b_{3}=-b_{2}$, we obtain $\alpha^{\prime}=\beta^{\prime}=0$, i.e., we have the algebra $A_{4}$.

Case 2.2. Let $\gamma \neq 0$. Then putting

$$
b_{2}=\frac{a_{1}^{2}}{\gamma}, \quad a_{3}=-\frac{a_{1}^{2} \alpha+a_{1} a_{2} \beta+a_{2}^{2} \gamma}{a_{1}}, \quad b_{3}=\frac{a_{1} b_{2} \beta+2 a_{2} b_{2} \gamma}{a_{1}},
$$

we get $\gamma^{\prime}=1, \alpha^{\prime}=\beta^{\prime}=0$, i.e., the algebra $A_{5}$ is obtained. 
Note that algebras with the conditions $(2,0,0),(1,0,0),(0,0,0)$ are associative algebras. Therefore, we can use the classification of four-dimensional algebras of Leibniz [1], i.e., choose algebras with the condition $A^{3}=0$.

Note that the problem of classification of complex five-dimensional Zinbiel algebras is open and the solution of this problem is equivalent to the classification of such associative algebras, which is still not obtained.

\section{References}

[1] S. Albeverio, B. A. Omirov, and I. S. Rakhimov. Classification of 4-dimensional nilpotent complex Leibniz algebras. Extracta Math., 21 (2006), 197-210.

[2] Sh. A. Ayupov and B. A. Omirov. On some classes of nilpotent Leibniz algebras. Sibirsk. Mat. Zh., 42 (2001), 18-29.

[3] A. S. Dzhumadil'daev. Identities for multiplications derived by Leibniz and Zinbiel multiplications. Abstracts of short communications of International conference "Operator algebras and quantum theory of probability" (2005), Tashkent, 76-77.

[4] A. S. Dzhumadil'daev and K. M. Tulenbaev. Nilpotency of Zinbiel algebras. J. Dyn. Control. Syst., 11 (2005), 195-213.

[5] J.-L. Loday. Cup-product for Leibniz cohomology and dual Leibniz algebras. Math. Scand., 77 (1995), 189-196.

[6] J.-L. Loday and T. Pirashvili. Universal enveloping algebras of Leibniz algebras and (co)homology. Math. Ann., 296 (1993), 139-158.

[7] J.-L. Loday, A. Frabetti, F. Chapoton, and F. Goichot. Dialgebras and Related Operads. Lecture Notes in Math., 1763, Springer-Verlag, Berlin, 2001.

[8] M. Vergne. Cohomologie des algèbres de Lie nilpotentes. Application à l'étude de la variété des algèbres de Lie nilpotentes. Bull. Soc. Math. France, 98 (1970), 81-116.

Received June 19, 2009

Revised October 06, 2009 